\title{
Previous Experience with Behavioral Control over Stress Blocks the Behavioral and Dorsal Raphe Nucleus Activating Effects of Later Uncontrollable Stress: Role of the Ventral Medial Prefrontal Cortex
}

\author{
José Amat, Evan Paul, Christina Zarza, Linda R. Watkins, and Steven F. Maier \\ Department of Psychology and Center for Neuroscience, University of Colorado, Boulder, Colorado 80309
}

Previous experience with stressors over which the subject has behavioral control blocks the typical behavioral consequences of subsequent exposure to stressors over which the organism has no behavioral control. The present experiments explored the involvement of the ventral medial prefrontal cortex (mPFCv) in mediating this "immunizing" or resilience producing effect of an initial experience with control. Behavioral immunization was blocked by inactivation of the mPFCv with muscimol at the time of the initial experience with control, as well as at the time of the later exposure to uncontrollable stress. Inhibition of protein synthesis within the mPFCv by anisomycin also blocked immunization when administered at the time of the initial controllable stress but had no effect when administered at the time of the later uncontrollable stress. Additional experiments found that the initial experience with control blocks the intense activation of serotonergic cells in the dorsal raphe nucleus that would normally be produced by uncontrollable stress, providing a mechanism for behavioral immunization. Furthermore, mPFCv activity during the initial controllable stressor was required for this effect to occur. These results suggest that the mPFCv is needed both to process information about the controllability of stressors and to utilize such information to regulate responses to subsequent stressors. Moreover, the mPFCv may be a site of storage or plasticity concerning controllability information. These results are consistent with recent research in other domains that explore the functions of the mPFCv.

Key words: medial prefrontal cortex; muscimol; anisomycin; stress; learned helplessness; dorsal raphe nucleus; serotonin; microdialysis; c-Fos

\section{Introduction}

The degree of behavioral control that an organism has over an aversive event potently modulates the impact of that event (Seligman and Maier, 1967; Weiss, 1968). For example, rats exposed to a series of uncontrollable inescapable tail shocks (IS) later fail to learn to escape from footshock in a shuttle box and show exaggerated fear conditioning and other aspects of potentiated anxiety for a $48 \mathrm{~h}$ period (for review, see Maier and Watkins, 1998). In contrast, subjects exposed to exactly equal amounts and distributions of controllable escapable tail shocks (ES) do not show these changes. These and related behavioral changes, which have been called "learned helplessness" (Maier and Seligman, 1976), have been linked to IS-induced changes in brainstem nuclei, most notably the serotonergic (5-HT) dorsal raphe nucleus (DRN) (Maier and Watkins, 2005) and the noradrenergic locus ceruleus (Weiss and Simson, 1986). With regard to the DRN, IS produces greater activation than does equated ES (Amat et al., 1998; Maswood et al., 1998; Grahn et al., 1999), and this activation is critical to the production of the behavioral effects of IS (Maier et al., 1995).

Received Jan. 10, 2006; revised Nov. 8, 2006; accepted Nov. 8, 2006.

This research was supported by National Institutes of Health Grant MH 50479.

Correspondence should be addressed to Dr. José Amat, Department of Psychology, University of Colorado, Campus Box 345, Boulder, C0 80309-0345. E-mail: amat@psych.colorado.edu.

DOI:10.1523/JNEUROSCI.3630-06.2006

Copyright $\odot 2006$ Society for Neuroscience ～0270-6474/06/2613264-09\$15.00/0
However, the DRN is a brainstem nucleus unlikely to detect whether a stressor is controllable, and thus DRN activation during stress is likely regulated by a "higher" structure. In this regard, Amat et al. (2005) recently reported that temporary inactivation of the ventral medial prefrontal cortex (mPFCv) with muscimol (M or MUS) during ES and IS eliminated the effects of control. Interestingly, inactivation of the $\mathrm{mPFCv}$ eliminated the difference by blocking the buffering effect of control rather than by reducing the impact of lack of control. That is, both ES and IS subjects with mPFCv inactivation showed similar intense DRN 5-HT activation and similar later behavioral deficits.

In a related but entirely different type of paradigm, the presence of control has been found to have the remarkable effect of changing the sensitivity of the organism to future stressful events, so-called "behavioral immunization" (Seligman and Maier, 1967). Thus, an experience of control over stress can blunt the behavioral impact of a future IS. More specifically, an initial experience with ES blocks the normal behavioral effects of subsequent IS, such as interference with shuttle-box escape behavior, even if the ES and IS experiences occur in different environmental contexts and at far different times (Seligman and Maier, 1967; Williams and Maier, 1977; Moye et al., 1981). These findings indicate that the experience of control is important in developing resilience.

Here we investigate changes in DRN 5-HT activity during IS in rats previously exposed to ES $7 \mathrm{~d}$ earlier and how any such proactive influence of ES is affected by inactivating the MPFCv. 
We also investigate whether the mPFCv is critical to the behavioral "immunizing" effect of previous control. Four questions are explored: does the mPFCv need to be engaged, (1) at the time of the original ES experience, (2) the period immediately after ES, or (3) during subsequent IS? Finally, is the MPFCv a site of plasticity for behavioral immunization?

\section{Materials and Methods}

In all experiments, rats were male Sprague Dawley rats (Harlan Sprague Dawley, Indianapolis, IN) weighing 275-350 g, housed four per cage on a $12 \mathrm{~h} \mathrm{light/dark} \mathrm{cycle} \mathrm{(lights} \mathrm{on} \mathrm{at} \mathrm{7:00} \mathrm{A.M.} \mathrm{and} \mathrm{off} \mathrm{at} \mathrm{7:00} \mathrm{P.M.).}$ Experiments were conducted between 8:00 A.M. and 4:00 P.M. All procedures were approved by the Institutional Animal Care and Use Committee of the University of Colorado at Boulder.

\section{Overall organization}

The present report contains experiments examining both the impact of an initial experience with a controllable and an uncontrollable stressor on the DRN activity and behavioral changes produced by a subsequent uncontrollable stressor. General features of methods will be described first, followed by description of the DRN methods, and then the behavioral methods.

\section{Surgery and cannulation}

Surgery was performed under anesthesia with $60 \mathrm{mg} / \mathrm{kg}$ sodium pentobarbital intraperitoneally. All rats were implanted with dual cannula guides for microinjections (26 gauge) with $1 \mathrm{~mm}$ center-to-center distance (Plastics One, Roanoke, VA). In most rats, the tips of the cannulas were aimed at the prelimbic/infralimbic (PL/IL) junction within the mPFCv: $2.2 \mathrm{~mm}$ rostral to bregma, $3.5 \mathrm{~mm}$ ventral from the dura mater, and $0.5 \mathrm{~mm}$ relative to midline. Some rats were implanted with cannula guides for microinjection $2 \mathrm{~mm}$ rostral (ventral orbital cortex, $n=4$ ) and $2 \mathrm{~mm}$ caudal (cingulate cortex area $2, n=4$ ) to the PL/IL regions as site-specificity control groups. For the microdialysis experiments, rats were implanted with a second cannula guide for microdialysis probes (CMA 12; CMA/Microdialysis, North Chelmsford, MA), with the tip terminating just above the caudal DRN: $8.3 \mathrm{~mm}$ caudal to bregma and 5 $\mathrm{mm}$ ventral from the dura matter at the midline (Amat et al., 2005). A screw cap of a $15 \mathrm{ml}$ conical centrifuge tube, whose central lid portion was removed, was also affixed to the skull so that its threads were exposed and it encircled the cannula guides. This was done so that the skull assembly could be protected during microdialysis. Rats were allowed to recover for 1-2 weeks before experimentation.

\section{Muscimol and anisomycin microinjection}

Animals were injected with $0.5 \mu \mathrm{l}$ of either $50 \mathrm{ng}$ of MUS (Sigma, St. Louis, MO), $100 \mathrm{ng}$ of anisomycin (A or ANI) (Sigma), or saline vehicle in each side of the mPFCv. Dual 33-gauge microinjectors (Plastics One) attached to polyethylene 50 tubing were inserted through the guides, from which they protruded $1 \mathrm{~mm}$. The other end of the tubing was connected to a $25 \mu \mathrm{l}$ Hamilton syringe that was attached to a David Kopf Instruments (Tujunga, CA) microinjection unit (model 5000). The volumes were injected over a period of $\sim 30 \mathrm{~s}$, and the injector was left in place for 2 min to allow diffusion. Any given injection was considered successful if fluid could be readily dispensed from the injector tips after it was withdrawn from the brain.

\section{Changes in 5-HT DRN activity}

\section{Behavioral and drug treatment}

Animals were subjected to two different treatments $7 \mathrm{~d}$ apart. During the first treatment, the animals received intra-mPFCv microinjection of MUS or vehicle (V or VEH). MUS- and VEH-injected rats either remained as home-cage controls (M/V-HC) or, $1 \mathrm{~h}$ after injection, were subjected to the IS/ES protocol described below in a separate room (M or $\mathrm{V}$-IS or V-ES). Thus, the design of the day 1 treatment was a 2 (MUS vs $\mathrm{VEH})$ by 3 (ES, IS, HC) factorial. Each ES and IS rat was placed in a Plexiglas box $(14 \times 11 \times 17 \mathrm{~cm})$ with a wheel mounted in the front and a Plexiglas rod extending from the back. The rats' tails were taped to the Plexiglas rod and affixed with copper electrodes. Rats received shocks in yoked pairs. An ES rat was in a box equipped with a wheel that, when turned by the paws as described below, terminated the tail shock to both rats. The other rat (IS) was in a box with a fixed wheel. The treatment consisted of 100 trials with an average intertrial interval of $60 \mathrm{~s}$. Shocks began simultaneously for both rats in a pair and terminated for both whenever the ES rat met a response criterion. Initially, the shock was terminated by a one-quarter turn of the wheel. The response requirement was increased by one-quarter turn when each of three consecutive trials was completed in $<5 \mathrm{~s}$. Subsequent latencies under $5 \mathrm{~s}$ increased the requirement by $50 \%$ up to a maximum of four full turns. If the requirement was not reached in $<30 \mathrm{~s}$, the shock was terminated and the requirement reduced to a single one-quarter turn. This procedure was used to ensure that the ES animals learned an operant response. Shock intensity was $1.0 \mathrm{~mA}$ for the first 30 trials, $1.3 \mathrm{~mA}$ for the second 30 trials, and $1.6 \mathrm{~mA}$ for the last 40 trials. We found this protocol to maintain good escape behavior in the ES subjects (Amat et al., 2005). The HC control rats were kept in Plexiglas boxes $(46 \times 20 \times 20 \mathrm{~cm})$ in a separate room, for all of the treatment groups.

The second treatment ( $7 \mathrm{~d}$ later) consisted of a session of IS (see below) during which microdialysis was performed for one set of six groups. Another set of six groups was killed $2 \mathrm{~h}$ after IS, and brains were processed for c-Fos and 5-HT immunohistochemistry. Thus, separate $3 \times 2$ factorial experiments were conducted to examine the DRN 5-HT and c-Fos responses to IS $7 \mathrm{~d}$ after previous ES, IS, or HC treatment, with and without mPFCv inactivation by MUS. There were seven or eight rats per microdialysis group and seven rats in each c-Fos group.

\section{In vivo microdialysis}

On the afternoon of the sixth day after the first treatment, a CMA 12 microdialysis probe $(0.5 \mathrm{~mm}$ in diameter, $1 \mathrm{~mm}$ membrane with a 20 $\mathrm{kDa}$ molecular weight cutoff; CMA/Microdialysis) was introduced through the cannula guide so that the membranous tip of the probe was within the DRN. A portion of a $15 \mathrm{ml}$ Eppendorf (Westbury, NY) tube was screwed onto the skull-mounted screw cap, through which the dialysis tubing, protected within a metal spring, entered and attached to the probe. Each animal was placed individually in a Plexiglas bowl (Bioanalytical Systems, West Layfayette, IN) and infused with isotonic Ringer's solution (Baxter, McGraw Park, IL) at a rate of $0.2 \mu \mathrm{l} / \mathrm{min}$ overnight. At 9:00 A.M. the next day, the flow rate was increased to $1.5 \mu \mathrm{l} / \mathrm{min}$ and a 90 min stabilization period was allowed. The infusion flow remained constant throughout the experiment. Samples were collected every $20 \mathrm{~min}$. After stabilization, four baseline samples were collected. Next the rats were placed in Plexiglas boxes that were designed to accommodate the dialysis tubing. Flow was not interrupted during the movement of the subjects. There they received 100 IS tail shocks ( $5 \mathrm{~s}$ duration each) at an average intertrial interval of $60 \mathrm{~s}$. Current intensity varied between 1 and $1.6 \mathrm{~mA}$ as described above. Five samples were collected during the shock session. After this, the rats were transferred back to the Plexiglas bowls in which three post-shock samples were collected.

5-HT concentration was measured in dialysates by HPLC with electrochemical detection. The system consisted of an ESA (Chelmsford, MA) 5600A Coularray detector with an ESA 5014B analytical cell and an ESA 5020 guard cell. The column was an ESA MD-150 (C-18, $150 \times 3.2$ $\mathrm{mm}$ ) maintained at $37^{\circ} \mathrm{C}$, and the mobile phase was the ESA buffer MD-TM. The analytical cell potentials were kept at -100 and $+220 \mathrm{mV}$ and the guard cell at $+250 \mathrm{mV}$. Dialysate $(27 \mu \mathrm{l})$ was injected with an ESA 542 autosampler that kept the dialysates at $6^{\circ} \mathrm{C}$. External standards (Sigma) were run each day to quantify 5 -HT.

\section{c-Fos and 5-HT immunohistochemistry}

Tissue preparation. Two hours after the last tail shock of the second treatment, the rats from these six groups were deeply anesthetized with sodium pentobarbital (Nembutal) and transcardially perfused with 100 $\mathrm{ml}$ of $0.9 \%$ saline containing $0.1 \%$ heparin followed by $250 \mathrm{ml}$ of $4 \%$ paraformaldehyde in 0.1 sodium phosphate buffer, $\mathrm{pH}$ 7.4. Brains were removed and postfixed in the same fixative overnight. After postfixation, brains were transferred to $30 \%$ sucrose and stored at $4^{\circ} \mathrm{C}$ until sectioning. 
Table 1. Experimental groups of the behavioral study and the procedure that they underwent on each of the 3 treatment days

\begin{tabular}{|c|c|c|c|c|}
\hline Group designation & Treatment 1 & Treatment 2 & Testing & Figures \\
\hline V-IS/IS & V-IS & IS & SHTLBOX & 4 \\
\hline V-ES/IS & V-ES & IS & SHTLBOX & 4 \\
\hline $\mathrm{V}-\mathrm{HC} / \mathrm{IS}$ & V-HC & IS & SHTLBOX & $4-8,10,11$ \\
\hline M-IS/IS & M-IS & IS & SHTLBOX & 4 \\
\hline M-ES/IS & M-ES & IS & SHTLBOX & 4 \\
\hline $\mathrm{M}-\mathrm{HC} / \mathrm{IS}$ & $\mathrm{M}-\mathrm{HC}$ & IS & SHTLBOX & 4 \\
\hline ES-V/IS & ES-V & IS & SHTLBOX & 5 \\
\hline ES-M/IS & ES-M & is & SHTLBOX & 5 \\
\hline IS/V-IS & IS & V-IS & SHTLBOX & 6 \\
\hline ES/V-IS & ES & V-IS & SHTLBOX & 6 \\
\hline IS/M-IS & IS & M-IS & SHTLBOX & 6 \\
\hline ES/M-IS & ES & M-IS & SHTLBOX & 6 \\
\hline M-ES/M-IS & M-ES & M-IS & SHTLBOX & 7 \\
\hline V-HC/V-HC & V-HC & $\mathrm{V}-\mathrm{HC}$ & SHTLBOX & $4-8,10,11$ \\
\hline ES/IS/V-SHTLBOX & ES & IS & V-SHTLBOX & 8 \\
\hline ES/IS/M-SHTLBOX & ES & IS & M-SHTLBOX & 8 \\
\hline$A-E S / I S$ & A-ES & IS & SHTLBOX & 10 \\
\hline$A-E S / A-I S$ & A-ES & A-IS & SHTLBOX & 10 \\
\hline ES-A/IS & ES-A & IS & SHTLBOX & 10 \\
\hline V-ES/A-IS & V-ES & A-IS & SHTLBOX & 11 \\
\hline
\end{tabular}

SHTLBOX, Shuttle box. If drug abbreviation precedes experimental treatment abbreviation, the drug was given before the treatment. If drug abbreviation follows experimental treatment abbreviation, the drug was given after the treatment.

Brains were rapidly frozen in $-40^{\circ} \mathrm{C}$ isopentane, and $35 \mu \mathrm{m}$ sections were obtained in a $-20^{\circ} \mathrm{C}$ cryostat. Free-floating sections were stored at $4^{\circ} \mathrm{C}$ in cryoprotectant until staining.

Immunohistochemistry. Immunohistochemical staining for c-Fos and 5-HT was conducted sequentially as described previously (Amat et al., 2005). Briefly, staining for c-Fos was conducted first using rabbit polyclonal Fos primary antibody (1:12,500; Santa Cruz Biotechnology, Santa Cruz, CA) and biotinylated goat anti-rabbit secondary antibody (Jackson ImmunoResearch, West Grove, PA) with 3,3'-diaminobenzidine (DAB) and nickel as chromogens. Staining for 5-HT used rabbit 5-HT primary antibody (1:10,000; ImmunoStar, Hudson, WI) and nonbiotinylated goat anti-rabbit $\operatorname{IgG}$ (Jackson ImmunoResearch) secondary antibody with peroxidase anti-peroxidase and $\mathrm{DAB}$ as chromogens.

Image analysis. Sections were assessed, by a blind observer, for the number of 5-HT-stained cells and the number of cells double-labeled for both 5-HT and c-Fos. c-Fos-stained nuclei were identified by dark brown or black ovoid particles. Larger reddish-tan particles, with or without Fos-stained nuclei, were counted as 5-HT-positive cells. Rostral DRN sections were comparable with an anteroposterior coordinate of -7.64 $\mathrm{mm}$ from bregma, middle DRN sections to $-8 \mathrm{~mm}$ from bregma, and caudal DRN sections to $-8.3 \mathrm{~mm}$ from bregma.

\section{Behavioral effects}

Animals in these experiments were subjected to three sequential experimental treatments. There were 20 groups directed at answering different questions. Rather than repeating control groups, the different groups were run in an intermixed manner, so that a particular control could be compared with multiple sets of groups to address more than one issue. During the first treatment, animals were subjected to either the ES/IS protocol as in the experiments above or remained as HC controls. Some groups received intra-mPFCv microinjection of either MUS or VEH $1 \mathrm{~h}$ before treatment as above or immediately after treatment. Other groups received intra-mPFCv ANI or VEH $1 \mathrm{~h}$ before or immediately after experimental treatment. The second treatment was performed $7 \mathrm{~d}$ later. Subjects received either IS in Plexiglas tubes or HC treatment. MUS, ANI, or VEH was microinjected $1 \mathrm{~h}$ before this treatment in some groups. Escape learning was tested in shuttle boxes $24 \mathrm{~h}$ after this second treatment (see below). VEH or MUS was microinjected $1 \mathrm{~h}$ before testing in some groups. Table 1 depicts the 20 experimental groups, the procedure that they received on each of the three treatment days, and the figures where their data are shown.

\section{First experimental treatment}

The rats were subjected to the same microinjections, IS/ES, and HC protocols described for the DRN experiments.

\section{Second experimental treatment}

The IS session was performed in Plexiglas tubes $17.5 \mathrm{~cm}$ long and $7.0 \mathrm{~cm}$ in diameter. The rat's tail extended from the rear of the tube and was taped to a Plexiglas rod. Rats received 100 IS tail shocks (5 s duration each) at an average intertrial interval of $60 \mathrm{~s}$. Current intensity varied between 1 and $1.6 \mathrm{~mA}$ as described above.

\section{Third experimental treatment}

Escape learning was performed in shuttle boxes measuring $46.0 \times 20.7 \times$ $20.0 \mathrm{~cm}$. Shock intensity was $0.6 \mathrm{~mA}$. The subjects were first given $5 \mathrm{~min}$ to adapt to the boxes. This was followed by two fixed ratio 1 (FR-1) escape trials at $1 \mathrm{~min}$ intervals. Here, the rat was required to cross to the other side of the box to terminate shock. Freezing was then scored for 20 min. The rats then received three additional FR-1 escape trials, followed by 25 FR- 2 trials that were analyzed as five blocks of five trials. Here, the rat was required to cross to the other side and then return to terminate shock. It is here that shuttle-box escape deficits occur after IS. Each shock terminated after $30 \mathrm{~s}$ if an escape response had not occurred. The freezing data will not be presented.

\section{Statistical analysis}

Data were analyzed by repeated-measures ANOVA followed by Fisher's protected least significant difference (PLSD) test post hoc comparison ( $\alpha$ set at 0.05$)$.

\section{Results}

Figure $1 A$ shows the cannula placements for both the mPFCv and site-specificity control microinjections, and Figure $1 B$ shows the microdialysis cannula placements.

\section{Changes in extracellular 5-HT in the caudal DRN during IS}

MUS had no effect on day 1 escape behavior in the wheel-turn boxes in this or the subsequent experiments. This has been extensively studied by Amat et al. (2005) and the data has been reported in detail and so will not be repeated here. Using in vivo microdialysis, we have shown previously that IS produces a large and persistent 5-HT efflux within the caudal DRN, whereas equal ES produces only a brief initial increase (Amat et al., 2005). Here we report the effect of IS on extracellular 5-HT levels in rats subjected to different experimental treatments $7 \mathrm{~d}$ earlier. Figure $2 \mathrm{~A}$ shows extracellular 5-HT during IS for the groups that received VEH on day 1 . The VEH and MUS groups that had received HC treatment on day 1 did not differ and so were pooled. The pooled group that had received HC on day 1 showed the usual large IS-induced increase in 5-HT efflux that persisted throughout and after IS. The dotted line shows the 5-HT efflux produced by ES from a previous study (Amat et al., 2005) for comparison. Previous IS on day 1 did not alter the DRN response to IS on day 8 . However, ES $7 \mathrm{~d}$ earlier dramatically reduced the 5-HT efflux produced by IS. Indeed, extracellular 5-HT was reduced to the level normally produced by ES. Figure $2 B$ shows extracellular levels of 5-HT for the groups given MUS before day 1 IS and ES, as well as the pooled day 1 HC control. MUS given before IS on day 1 had no effect on the 5-HT response to IS on day 8 . As was the case for the IS group given VEH on day 1 , IS on day 8 produced a large increase in 5 -HT that did not differ from the IS-induced 5-HT levels in the HC controls. However, MUS had a robust effect when given before ES. MUS administered before ES completely eliminated the reduction in IS-induced 5-HT produced by the day 1 ES. ANOVA on baseline samples taken before the stressor showed no differences between any of the groups (all 


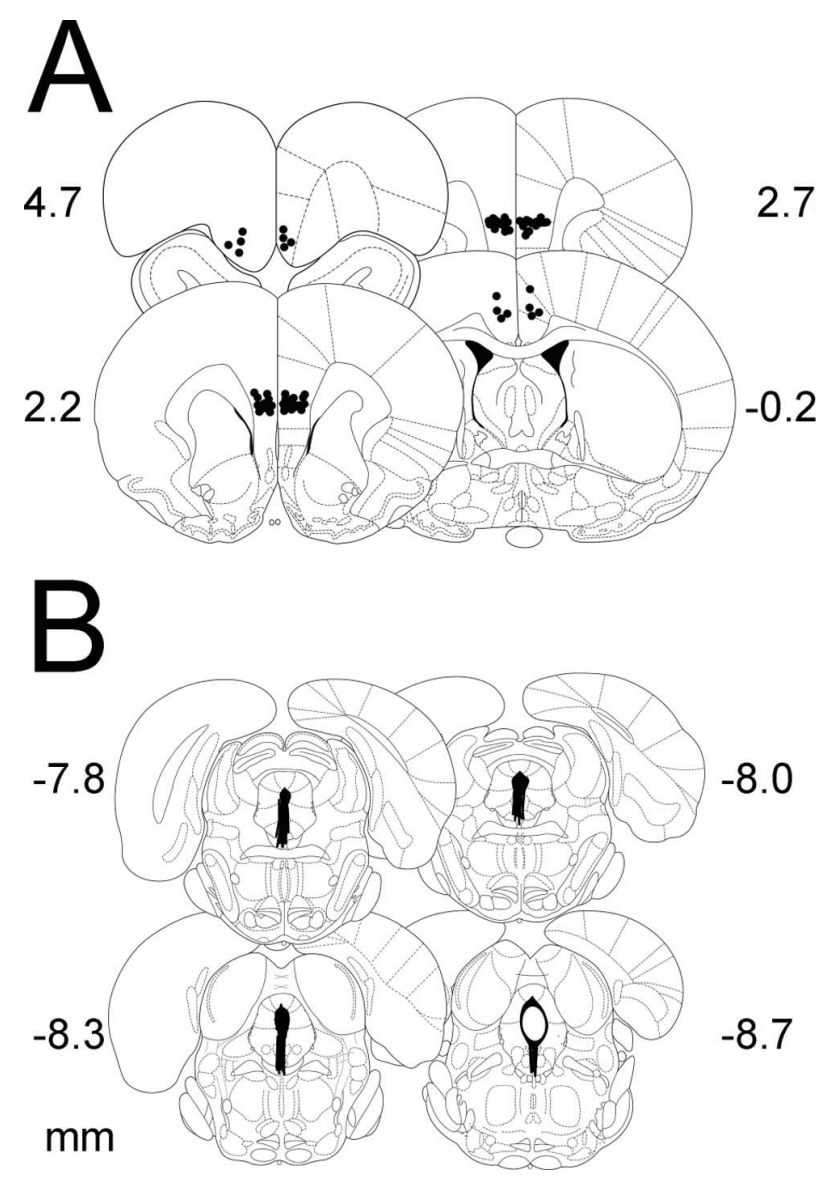

Figure 1. A, Microinjection cannula placements. The black circles represent the sites of the injection cannula tips. $\boldsymbol{B}$, Microdialysis probe placements. Black bars represent the placement of microdialysis probes. Numerals indicate distance from bregma (in millimeters).

$F$ values $<1.0)$. ANOVA during the stressor showed significant effects of stress condition (IS, ES, and HC) $\left(F_{(4,128)}=5.878 ; p<\right.$ $0.0002)$ and the interaction between stress condition and MUS treatment $\left(F_{(4,32)}=3.265 ; p<0.023\right)$. Fisher's PLSD test indicated that $\mathrm{V}$-ES/IS is different from the other five groups, which did not differ among themselves. ANOVA conducted on poststress samples did not show significant effects of stress condition or the interaction between stress condition and MUS treatment.

\section{c-Fos expression in 5-HT-labeled neurons in the DRN}

We found previously that IS produces greater activation of DRN 5-HT neurons than equated ES as assessed by c-Fos protein expression in 5-HT-labeled neurons (Grahn et al., 1999). This effect of controllability was especially prominent in middle and caudal regions of the DRN. Here we examined whether previous IS or ES would alter this pattern and whether the mPFCv is critical to any such effects. Figure $3 A-C$ shows micrographs of DRN coronal sections $(-8 \mathrm{~mm}$ from bregma) that show typical c-Fos and 5-HT labeling in V-ES/IS, V-IS/IS, and M-ES/IS subjects. Figure 4 shows the percentage of 5-HT-labeled cells that express c-Fos protein in the caudal and middle DRN after day 8 IS. Fos expression did not differ in the MUS and VEH HC day 1 groups, and so these groups were pooled. It should be noted that previous work (Grahn et al., 1999; Takase et al., 2004; Amat et al., 2005) has indicated that there is virtually no Fos expression in 5-HT-labeled DRN neurons in home-cage control subjects. Thus, it can be presumed that the Fos observed was induced by the IS. Previous day 1 IS had no effect on day 8 IS-induced Fos, but previous ES
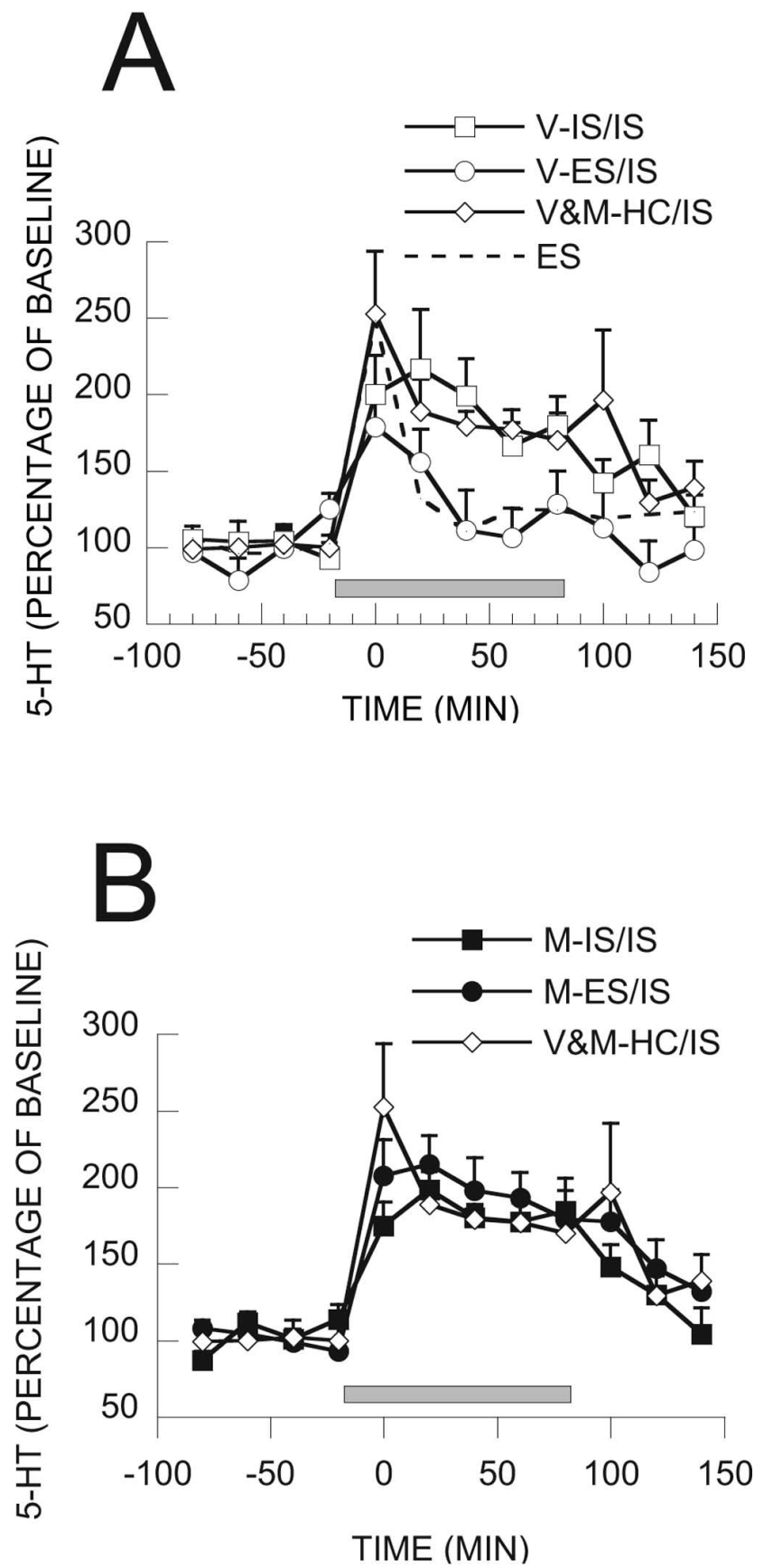

Figure 2. $\quad \boldsymbol{A}, 5-\mathrm{HT}$ as percentage of baseline in caudal DRN during IS from groups of rats receiving VEH and different stress treatments $7 \mathrm{~d}$ earlier. The dotted line represents 5 - $\mathrm{HT}$ in rats exposed to ES from a previous study, for comparative purposes. Squares received previous V-IS, circles received previous V-ES, and diamonds are pooled data of previous $V$ or M-HC. $B, 5-H T$ as percentage of baseline in caudal DRN during IS from groups of rats receiving MUS and different stress treatments 7 d earlier. Black squares received previous M-IS, black circles received previous M-ES, and diamonds are pooled data of previous V or M-HC. The gray bar represents the time of stressor exposure in the wheel-turn boxes in $\boldsymbol{A}$ and $\boldsymbol{B}$.

reduced the Fos expression observed. Furthermore, inactivation of the mPFCv during day 1 ES blocked this proactive effect of ES. ANOVA showed significant effects of stress condition $\left(F_{(2,29)}=\right.$ $5.145, p<0.02$ in caudal DRN; and $F_{(2,29)}=4.27, p<0.03$ in middle DRN) and the interaction between stress condition and MUS $\left(F_{(2,29)}=7.213, p<0.003\right.$ in caudal DRN; and $F_{(2,29)}=$ $4.936, p<0.02$ in middle DRN). Fisher's PLSD post hoc comparison showed that the $\mathrm{V}$-ES group differed from the other groups, 
which did not differ from each other, in both regions of the DRN. Thus, previous ES reduced the increased c-Fos expression in caudal and middle DRN 5-HT neurons after subsequent IS. There was no effect of stressor controllability or MUS in rostral DRN (data not shown).

\section{Role of the mPFCv in} behavioral immunization

Intra-mPFCv MUS administered before treatment during the "immunization phase" (day 1 treatment)

Here the major question was whether in-

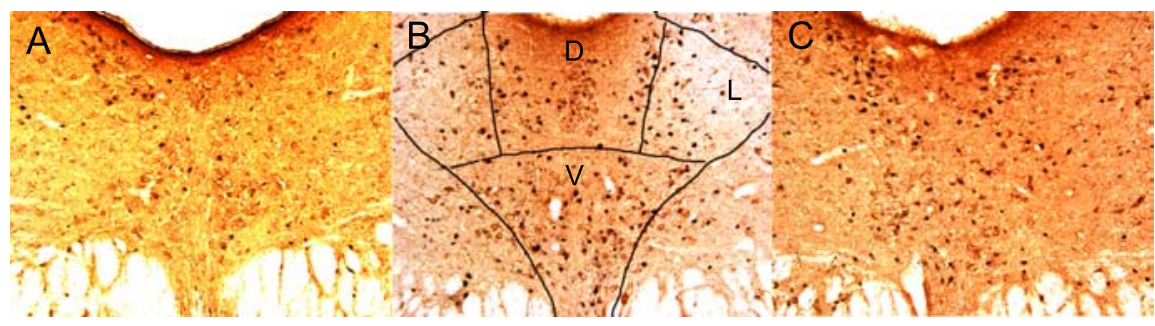

Figure 3. Photomicrographs ( $20 \times)$ of coronal DRN sections at $-8 \mathrm{~mm}$ from bregma ( $D$, dorsal; $V$, ventral; $L$, lateral) of rats exposed to IS ending $2 \mathrm{~h}$ before death. The section in $A$ is from a subject microinjected with VEH into the $\mathrm{VPFCm}$ and exposed to ES $7 \mathrm{~d}$ earlier. In $\boldsymbol{B}$, a similar microinjection was made, and the subject was exposed to IS. In $\boldsymbol{C}$, the subject received a microinjection of MUS followed by ES. The sections were double labeled for c-Fos (small black particles) and serotonin (larger brown-reddish particles).

activation of the mPFCv during the immu-

nization phase would interfere with the ability of ES to block the behavioral effects of IS. Figure 5 shows the FR-2 shuttle-box latencies for the relevant groups. Because the trials automatically terminated after $30 \mathrm{~s}$, mean latencies near $30 \mathrm{~s}$ indicate that most subjects in that group failed to escape. As is typical, IS produced very poor escape behavior $24 \mathrm{~h}$ later. This is shown by group $\mathrm{V}-\mathrm{HC} / \mathrm{IS}$, which received only a vehicle injection during treatment 1 and then IS $7 \mathrm{~d}$ later during treatment 2. Shuttle-box testing occurred $24 \mathrm{~h}$ later. Again as is typical, ES during treatment 1 blocked the effects of later IS (V-ES/IS). V-HC/V-HC serves as a baseline, and, as is evident, group V-ES/IS performed similarly to this baseline group. This immunizing effect is attributable to the escapability of the shock in the initial session because an initial treatment with IS (V-IS/IS) did not reduce the interference with escape produced by later IS. Indeed, an initial exposure to IS produced even poorer escape. Importantly, MUS given before ES (MUS-ES/IS) completely eliminated the immunizing effect of ES, that is, ES did now not block or reduce the effects of later IS. MUS administration before IS or HC on treatment 1 had no effect. These conclusions are confirmed by ANOVA. The effects of groups $\left(F_{(6,48)}=3.80 ; p<0.004\right)$, trial blocks $\left(F_{(4,192)}=13.61 ; p<0.0001\right)$, and the interaction of groups and trial blocks $\left(F_{(24,192)}=3.98 ; p<0.0001\right)$ were significant. Subsequent Fisher's PLSD tests (0.05) indicated that $\mathrm{V}-\mathrm{HC} / \mathrm{V}-\mathrm{HC}$ and V-ES/IS differed from all of the other groups, which did not differ among themselves. V-HC/V-HC and VES/IS did not differ from each other.

\section{Intra-mPFCv MUS administered after treatment during the immunization phase (day 1 treatment)}

Here the question was whether intra-mPFCv MUS given after ES would similarly block the immunizing effects of ES. Figure 6 shows the results from the groups designed to address this question. With regard to escape behavior, MUS given after ES (ES-M/ IS) did not substantially reduce the immunizing effects of ES (ES-V/IS). The effects of groups $\left(F_{(3,27)}=6.59 ; p<0.002\right)$ and the interaction of groups and trial blocks $\left(F_{(12,108)}=2.81 ; p<\right.$ 0.003 ) were significant. Fisher's PLSD test indicates that V-HC/IS differed from all of the other groups, which did not differ among themselves.

Intra-mPFCv MUS administered before IS at treatment 2 (day 8) Here the critical question was whether intra-mPFCv MUS at the time of IS would reduce or eliminate the subject's ability to use the previous ES experience. Figure 7 shows the data from the relevant groups. As above, previous ES blocked the interfering effect of IS, and this was not altered by vehicle injection before IS (ES/V-IS). However, animals given MUS before IS were no longer protected by previous ES (ES/M-IS). These subjects es-
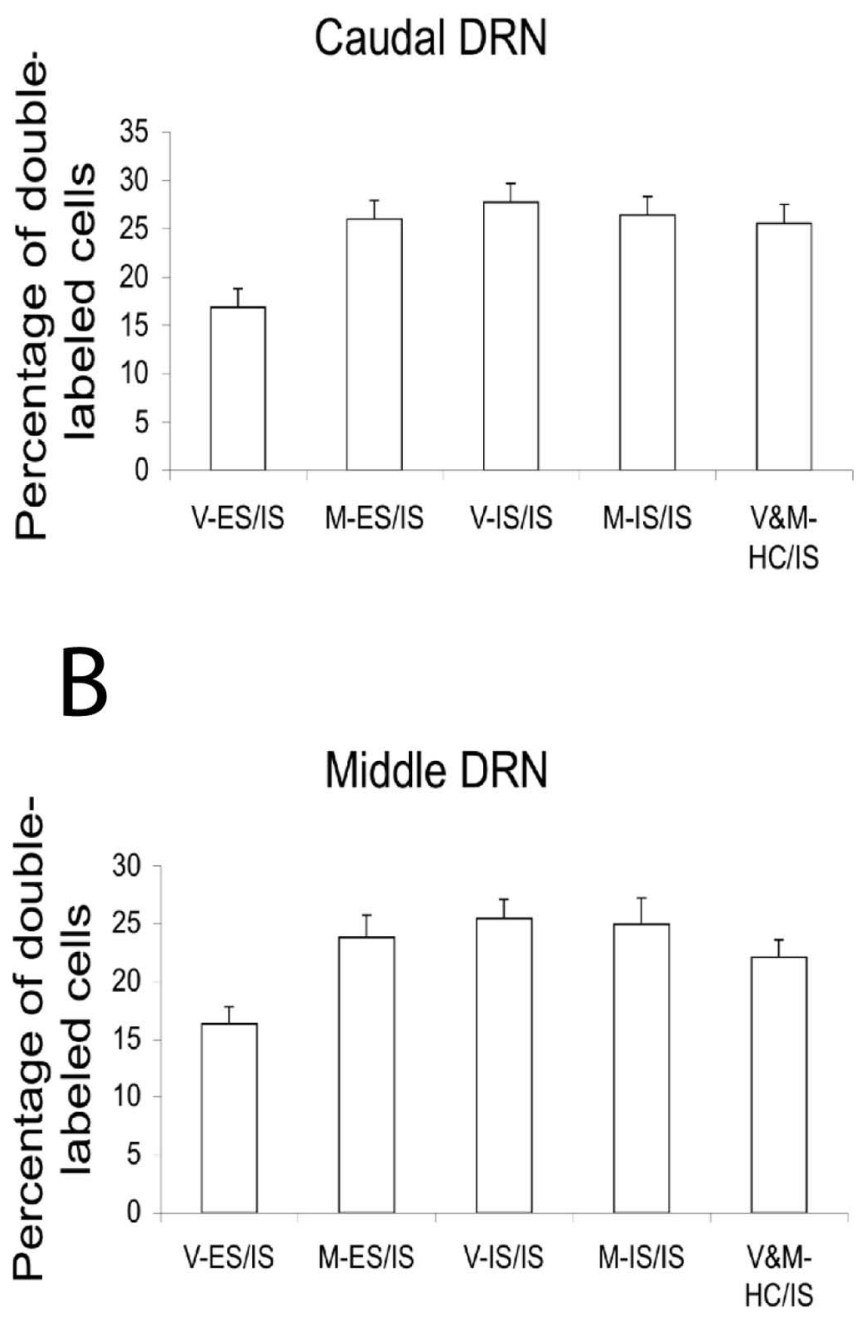

Figure 4. Percentage of 5-HT-labeled cells expressing c-Fos in caudal $(\boldsymbol{A})$ and middle $(\boldsymbol{B})$ DRN. All groups received IS ending $2 \mathrm{~h}$ before death, and either VEH or MUS and ES, IS, or HC $7 \mathrm{~d}$ earlier, as indicated below each bar.

caped as poorly as those given either no treatment or IS on treatment 1. ANOVA yielded reliable effects of groups $\left(F_{(5,42)}=5.92\right.$; $p<0.0003)$, trial blocks $\left(F_{(4,20)}=9.43 ; p<0.0001\right)$, and the interaction of groups and trail blocks $\left(F_{(20,168)}=2.94 ; p<\right.$ 0.0001). Fisher's PLSD test indicates that V-HC/V-HC and ES/ 


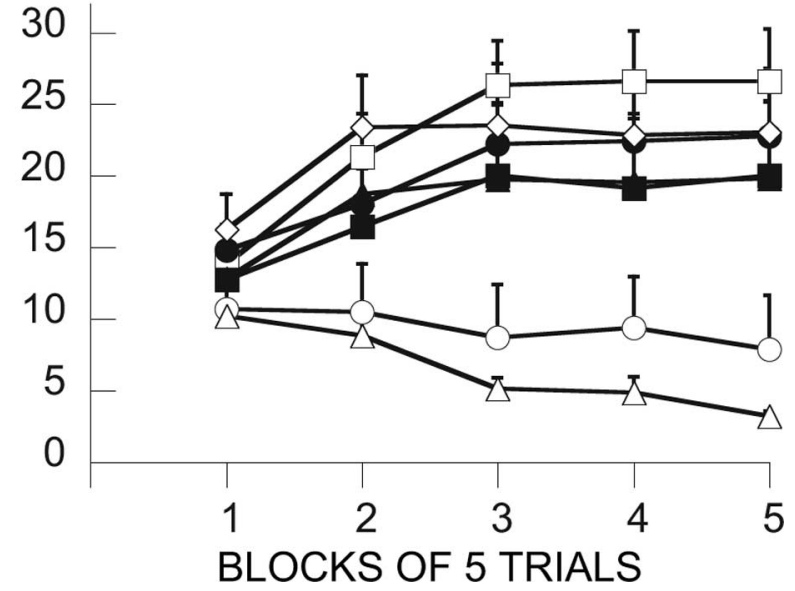

Figure 5. Effect of muscimol administration in the $\mathrm{VPFCm}$ before the immunization phase treatment (treatment 1). The graph in this figure and in Figures $6-9,11$, and 12 , shows the mean shuttle-box escape latencies across blocks of five shuttle-box FR-2 escape trials (third experimental treatments). $\square$, V-IS/IS; $\mathbf{\square}, \mathrm{M}-\mathrm{IS} / \mathrm{IS} ; \bigcirc, \mathrm{V}-\mathrm{ES} / \mathrm{IS} ; \boldsymbol{\bullet}, \mathrm{M}$-ES/IS; $\diamond, \mathrm{V}-\mathrm{HC} / \mathrm{IS} ; \boldsymbol{\Delta}$ $\mathrm{M}-\mathrm{HC} / \mathrm{IS} ; \triangle, \mathrm{V}-\mathrm{HC} / \mathrm{V}-\mathrm{HC}$ (for an explanation of the treatment group abbreviations, see Table 1).

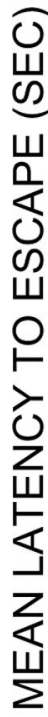

30

U
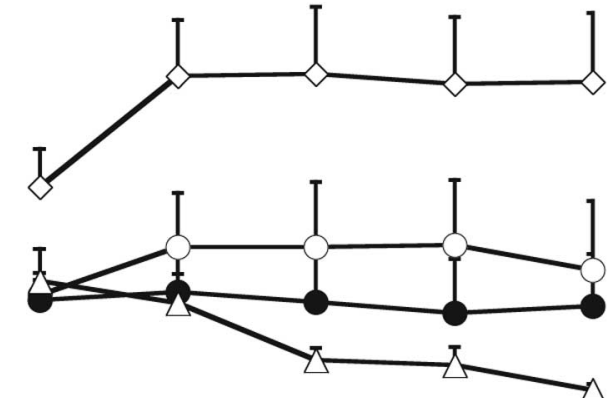

3

4

5

\section{BLOCKS OF 5 TRIALS}

Figure 6. Shuttle-box escape latencies showing the effect of intra-vPFCm muscimol administration given after the immunization phase treatment. $\bigcirc, \mathrm{ES}-\mathrm{V} / \mathrm{IS} ; \bigcirc, \mathrm{ES}-\mathrm{M} / \mathrm{IS} ; \diamond, \mathrm{V}-\mathrm{HC} / \mathrm{IS}$; $\triangle, \mathrm{V}-\mathrm{HC} / \mathrm{V}-\mathrm{HC}$.

V-IS differ from all of the other groups, which do not differ among themselves.

Intra-mPFCv MUS administered before both ES at treatment 1 and IS at treatment 2

The issue addressed here is whether the elimination of the effects of ES by MUS administered at either treatment 1 or treatment 2 could be explained by a state dependency (Castellano and McGaugh, 1990). As Figure 8 shows, administration of MUS at both times still eliminated the immunizing effects of ES; therefore, the effect of MUS at either treatment 1 or treatment 2 cannot be explained as resulting from a state dependency. The effects of groups $\left(F_{(2,21)}=7.72 ; p<0.004\right)$, and the interaction between groups and trial blocks $\left(F_{(8,84)}=3.81 ; p<0.0007\right)$ were signifi-

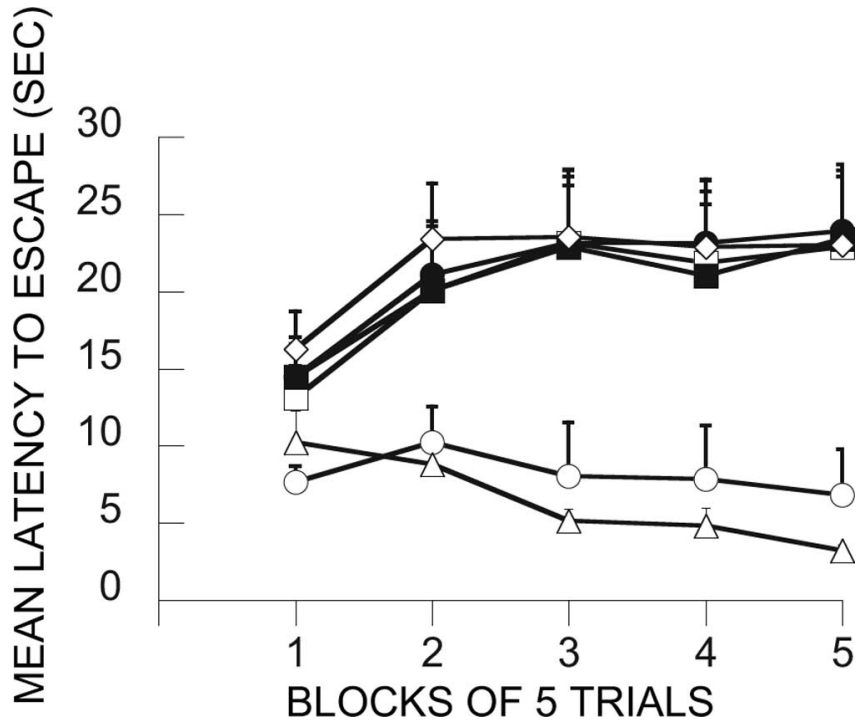

Figure 7. Shuttle-box escape latencies showing the effect of $\mathrm{vPFCm}$ muscimol administration before IS at treatment 2. $\square$, IS/V-IS; $\mathbf{\square}, \mathrm{IS} / \mathrm{M}-\mathrm{IS} ; \mathrm{O}, \mathrm{ES} / \mathrm{V}-\mathrm{IS} ; \boldsymbol{\bullet}, \mathrm{ES} / \mathrm{M}-\mathrm{IS} ; \diamond, \mathrm{V}-\mathrm{HC} / \mathrm{IS}, \mathbf{\Delta}$, $\mathrm{V}-\mathrm{HC} / \mathrm{V}-\mathrm{HC}$
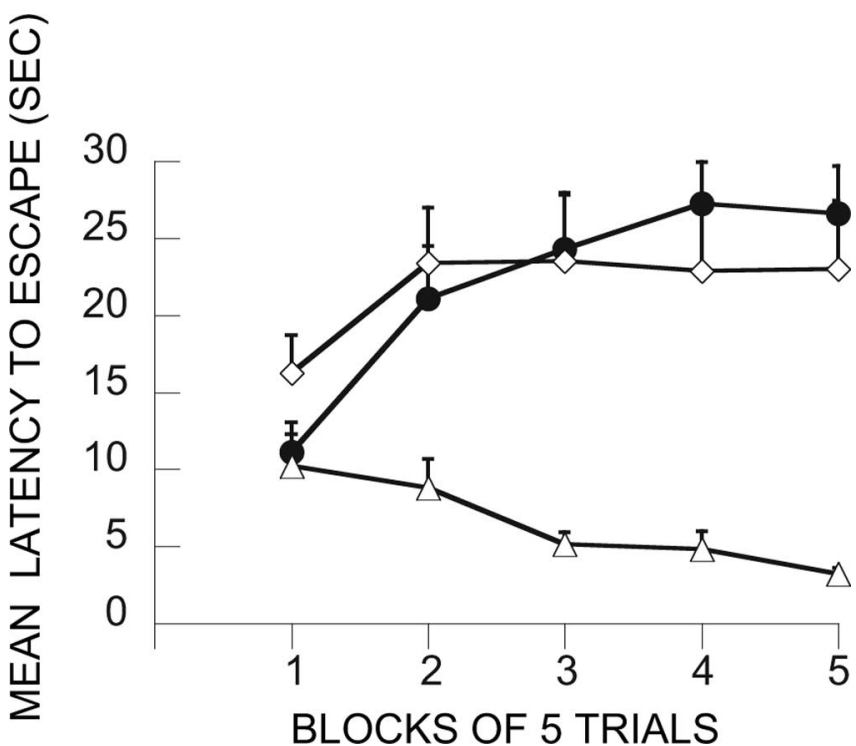

Figure 8. Shuttle-box escape latencies showing the effect of vPFCm muscimol administration before both ES at treatment 1 and IS at treatment 2. $\mathrm{M}$-ES/M-IS; $\diamond, \mathrm{V}-\mathrm{HC} / \mathrm{IS} ; \triangle$, $\mathrm{V}-\mathrm{HC} / \mathrm{V}-\mathrm{HC}$.

cant. V-HC/V-HC differed from the other two groups, which did not differ between themselves.

Intra-mPFCv MUS administered before shuttle-box testing (day 9)

Figure 9 contains the data for the groups designed to determine whether MUS delivered at the time of shuttle-box testing would influence the ability of ES on treatment 1 to block the effects of later IS on behavior. As is evident, MUS delivered before shuttlebox testing (ES/IS/M-T) did not influence the immunizing effect of the initial ES. With regard to escape behavior, the effect of groups $\left(F_{(3,28)}=7.84\right)$ and the interaction between groups and trial blocks $\left(F_{(12,112)}=2.19 ; p<0.02\right)$ are reliable. Fisher's PLSD test indicates that $\mathrm{V}$-HC/IS differs from the others, which do not differ among themselves. 


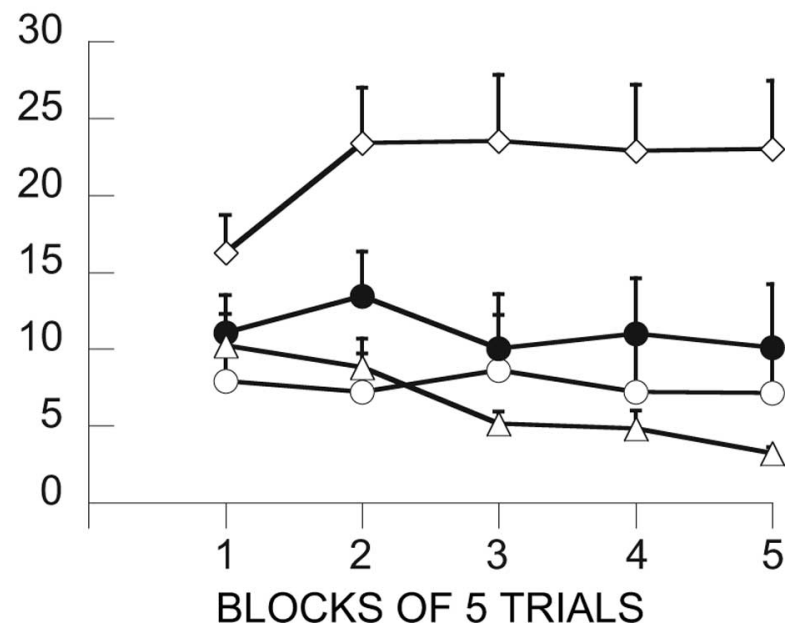

Figure 9. Shuttle-box escape latencies showing the effect of vPFCm muscimol administration before shuttle-box testing. $\mathrm{O}, \mathrm{ES} / \mathrm{IS} / \mathrm{V}-\mathrm{T} ; \bullet, \mathrm{ES} / \mathrm{IS} / \mathrm{M}-\mathrm{T} ; \diamond, \mathrm{V}-\mathrm{HC} / \mathrm{S} ; \triangle \mathrm{V}, \mathrm{V}-\mathrm{HC} / \mathrm{V}-\mathrm{HC}$.

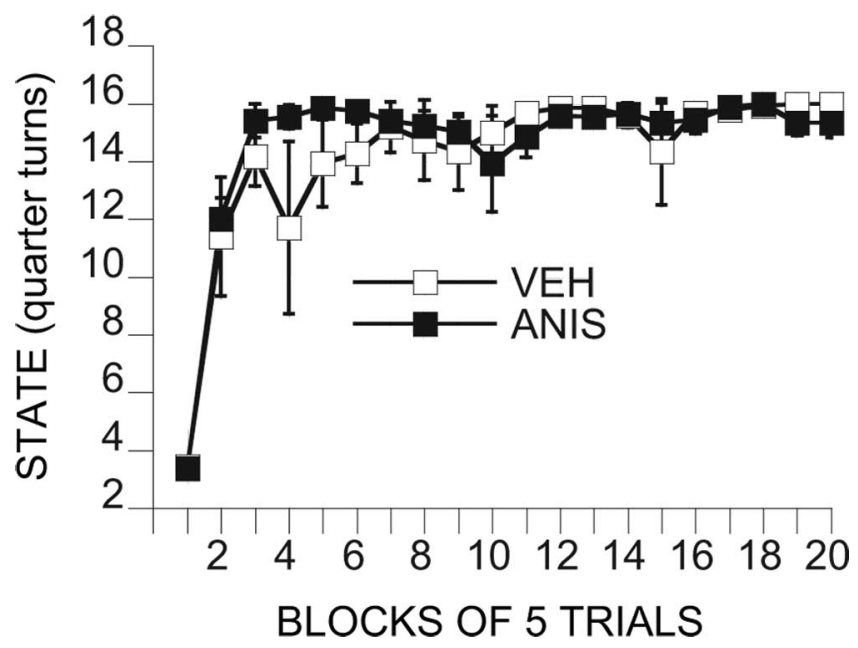

Figure 10. Efficiency of wheel-turn escape behavior during exposure to controllable tail shock in subjects that had received $\mathrm{VPFC}$ anisomycin (ANIS) or vehicle (VEH) 60 min before the shock session. The "state" is the mean number of one-quarter turns of the wheel attained as the escape requirement on each trial.

Intra-mPFCv ANI before ES at treatment 1 (day 1) or before IS at treatment 2 (day 8 )

Because we have not examined ANI before, it was possible that ANI would influence escape learning during treatment 1 . Figure 10 depicts a measure of escape efficiency across the 100 training trails. As described in Materials and Methods, the number of turns of the wheel required to escape was increased as the subjects escaped more rapidly, with a maximum of 16 one-quarter turns required. That is, animals that were responding rapidly were required to make 16 one-quarter turns to terminate each shock, whereas animals that responded more slowly had reduced requirements. Clearly, ANI did not interfere with escape performance. Figure 11 shows the shuttle-box latencies for the relevant groups. ANI administered before ES (A-ES/IS) eliminated the ability of ES to block the interfering effects of later IS (V-ES/IS). ANI given before IS and ES did not reduce the impact of ANI, eliminating the possibility of a state dependency. In contrast to muscimol, ANI delivered after ES (ES-A/IS) did have some influ-

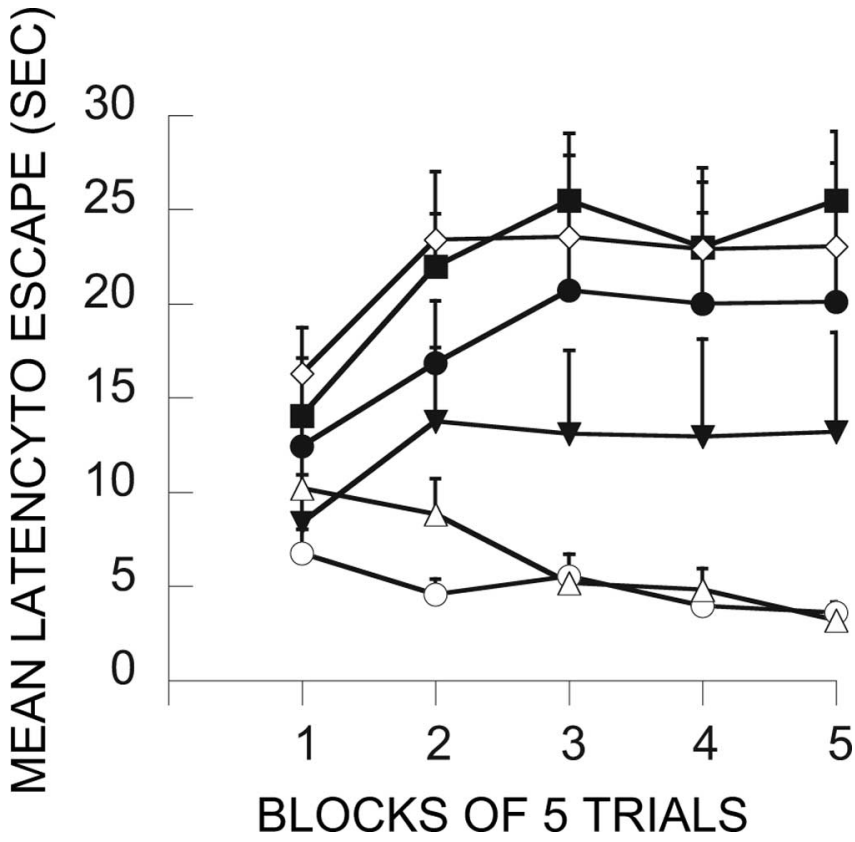

Figure 11. Shuttle-box escape latencies showing the effect of vPFCm anisomycin administration before $\mathrm{ES}$ at treatment 1 or IS at treatment $2 . \mathrm{O}, \mathrm{V}-\mathrm{ES} / \mathrm{IS} ; \boldsymbol{\bullet}, \mathrm{A}-\mathrm{ES} / \mathrm{IS} ; \bullet, \mathrm{A}-\mathrm{ES} / \mathrm{A}-\mathrm{IS} ; \boldsymbol{\nabla}$, ES-A/IS; $\diamond, V-H C / S$; $\triangle$, V-HC/V-HC.

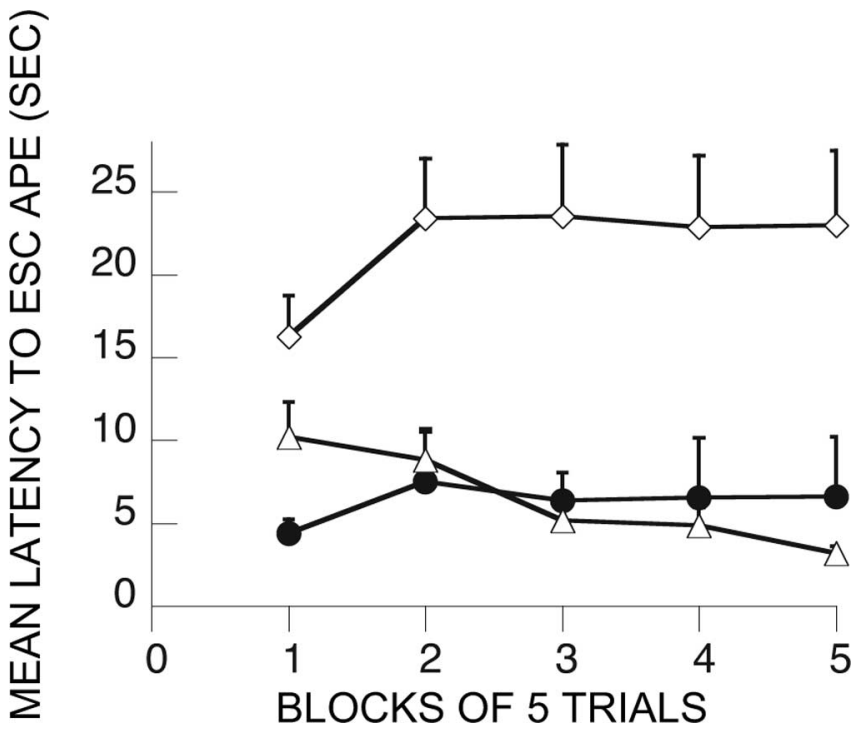

Figure 12. Shuttle-box escape latencies showing the effect of $\mathrm{vPFCm}$ anisomycin before is at treatment 2., $\mathrm{V}$-ES/A-IS; $\diamond, \mathrm{V}-\mathrm{HC} / \mathrm{IS} ; \triangle, \mathrm{V}-\mathrm{HC} / \mathrm{V}-\mathrm{HC}$.

ence, reducing but not eliminating the immunizing properties of ES. ANOVA yields significant effects of groups $\left(F_{(5,42)}=7.48\right.$; $p<0.0001)$, trial blocks $\left(F_{(4,168)}=7.07 ; p<0.00001\right)$, and the interaction of groups and trial blocks $\left(F_{(20,168)}=3.68 ; p<\right.$ $0.0001)$. Groups V-HC/V-HC and V-ES/IS differed significantly from all of the other groups but not between themselves. In addition, ES-A/IS differed from A-ES/A-IS and V-HC/IS.

Intra-mPFCv ANI before IS at treatment 2 (day 8)

In contrast to muscimol, ANI administered before IS did not reduce the immunizing effects of previous ES (Fig. 12). The effect of groups $\left(F_{(2,21)}=13.45 ; p<0.0002\right)$ and the interaction between groups and trial blocks $\left(F_{(8,84)}=4.62 ; p<0.0001\right)$ were 
significant. V-HC/IS differed from the other two groups, which did not differ between each other.

\section{Site placement controls}

Four rats were injected with MUS within the ventral orbital cortex and four within the cingulate cortex $1 \mathrm{~h}$ before a session of ES (treatment 1). Seven days later, they received a session of IS (treatment 2). The two groups were pooled together because they showed similar immunizing effects, which did not differ from that observed in the V-ES/IS group (data not shown). Thus, MUS injected $2 \mathrm{~mm}$ rostral or $2 \mathrm{~mm}$ caudal to the IL/PL regions did not prevent the immunizing effect of ES.

\section{Discussion}

The research reported here is the first to explore the neural mechanisms mediating how an initial experience with a controllable stressor can block the later behavioral effects of an uncontrollable stressor. The experiments were based on two previous sets of findings. The first is that IS, compared with equal ES, induces a greater activation of caudal DRN 5-HT neurons (Amat et al., 1998; Maswood et al., 1998; Grahn et al., 1999) and that this activation is critical to producing later behavioral changes such as escape failure (Maier et al., 1995). Thus, here we explored whether a previous experience with ES, although $7 \mathrm{~d}$ earlier, might reduce the DRN activation produced by IS as measured by both 5-HT efflux and c-Fos expression in 5-HT-labeled neurons within the caudal DRN.

Remarkably, the activating effect of IS on the DRN proved to depend on previous stress experience. Exposure to ES $7 \mathrm{~d}$ earlier dramatically reduced both IS-induced 5-HT efflux and c-Fos expression in 5-HT-labeled cells within the caudal DRN. Indeed, previous ES reduced the effects of IS to the magnitude typical of a first ES experience, despite the fact that the rat did not have control over the stressor. That is, if the subjects first experience was with ES, the DRN responded to the IS as if it were controllable. These data further support a critical role for DRN activation in producing the behavioral consequences of IS. Previous experience with ES is known to block the typical behavioral effects of IS, and here previous ES blocked the DRN activation produced by IS.

The second set of previous findings was that activation of the $\mathrm{mPFCv}$ during ES is necessary for ES failure to activate DRN neurons and the failure of ES to produce the behavioral changes typical of only IS (Amat et al., 2005). Thus, here we explored the impact of $\mathrm{mPFCv}$ inactivation in the immunization paradigm.

With regard to the behavioral consequences of IS, we examined the impact of intra-mPFCv MUS at both the time of the initial experience with control and the time of the later uncontrollable stressor. In terms of inactivation at the time of IS, intramPFCv MUS during IS prevented the previous ES experience from exerting its usual protective effect. The question here was whether the mPFCv is necessary to use the information that the stressor had been controllable in the past to modulate the impact of the uncontrollable stressor. Although the mPFCv had been available during and after the initial experience with control, intra-mPFCv MUS during the later uncontrollable stressor eliminated the buffering effect of the initial control experience. Thus, the $\mathrm{mPFCv}$ is involved in the use of the previous experience with control to modulate the impact of the later uncontrollable stressor. For a buffering effect to occur, it is not enough that the organism has learned the controlling response and consolidated a memory of this experience. Clearly, the mPFCv is involved in using this memory, to modulate the processes that underlie the production of the behavioral sequelae of the uncontrollable stress experience.
It was possible that, although mPFCv function is necessary to use the previous control experience, the mPFCv might not be needed at the time of the initial ES. This is because intra-mPFCv MUS does not interfere with the acquisition of the wheel-turn escape response (Amat et al., 2005). However, basic associative learning and more complex aspects of cognition are subserved by different systems (Robbins, 2000; Cardinal et al., 2002). The mPFCv has generally been found to not be involved in basic association or habit learning (Yin et al., 2004) but rather to perform more complex "executive functions" (Miller and Cohen, 2001), and the issue here was whether these higher-level functions are required at the time of the ES to allow it to exert its proactive protection later. The results were clear. Inactivation of the mPFCv during the ES session eliminated the proactive effects of control, although it did not interfere with the wheel-turn escape response. It is important to note that MUS delivered immediately after the ES session was without effect, and so it is $\mathrm{MPFCV}$ activity during the stress session that is critical. Thus, the present data are consistent with Amat et al. (2005) in suggesting that the mPFCv is not the site of escape learning (Graeff et al., 1996) but rather is the structure that uses signals of the presence of control during the stress experience to modulate the neural systems on which uncontrollable stress acts to produce escape failure, exaggeration of fear conditioning, etc.

The role of the mPFCv at the time of the uncontrollable stress is likely to involve the use of information that the stressor had been controllable in the past, to modulate the effects of the uncontrollable stressor on neural structures that mediate the behavioral sequelae of the stressor. However, the role of the mPFCv at the time of the original ES is less certain. The experiments reported by Amat et al. (2005) indicate that the mPFCv detects that the stressor is controllable and then regulates the activity of the DRN and perhaps other structures accordingly. The MUS data reported here are consistent with this conclusion. However, it is possible that the mPFCv might also be a site of plasticity or storage of this information. This issue is often investigated by determining whether protein synthesis in the structure in question is necessary for subsequent memory (Inda et al., 2005). ANI has been most often used as an agent to block protein synthesis (Santini et al., 2004), and, in the present experiments, intra-mPFCv ANI delivered before ES eliminated the buffering effect of control, suggesting that the mPFCv might indeed be a site at which information about control is maintained.

However, ANI has many effects other than inhibition of protein synthesis (Ogawa et al., 2004), and it has been argued that caution should be used in inferring that interference with memory by ANI indeed implicates a protein synthesis-dependent process (Routtenberg and Rekart, 2005). Because many of these other effects of ANI involve neural depressant or inhibitory processes (Flexner and Goodman, 1975), it is interesting to compare the effects of ANI with those of muscimol. Indeed, the pattern of results was quite different. Both were effective when given before ES, but MUS was without impact when administered after ES, and ANI was without effect when given before the IS. Thus, the impact of the ANI could not have been produced by simple inhibition of neural function and perhaps was produced by its ability to inhibit protein synthesis.

The studies that were conducted examining the role of the mPFCv in the immunizing effects of ES on subsequent ISinduced DRN activation, rather than behavior, examined only mPFCv inactivation during the initial ES. Intra-mPFCv MUS before ES (day 1) blocked the immunizing effect of this ES exposure. That is, IS $7 \mathrm{~d}$ later produced its usual large 5-HT efflux and 
c-Fos expression in 5HT-labeled neurons within the caudal DRN, when the mPFCv is inhibited by MUS during previous ES. Previous work indicates that pyramidal cells of the MPFCv inhibit the activation of DRN 5-HT cells during stress if the stressor is controllable. This can be accomplished through the glutamatergic projection from the mPFCV (Peyron et al., 1998) that synapses preferentially onto GABAergic interneurons within the DRN (Jankowski and Sesack, 2004). It is thus possible that, during IS, the memory of the previous controllable experience leads the mPFCv to inhibit the DRN, although the stressor is uncontrollable. In essence, this would amount to something like an "illusion of control."

The present results fit well with other recent research directed at understanding the role of the $\mathrm{mPFCv}$ in stress-related processes. For example, lesions of the mPFCv have no effect on the acquisition or the simple extinction of conditioned fear (Morgan et al., 1993) but interfere with the organism's memory of extinction (Quirk et al., 2000) or its ability to use information about extinction to guide behavior (Sotres-Bayon et al., 2004). Within the immunization paradigm explored here, the results can also be viewed as implicating a role for the MPFCv in using information, here about the presence of control, to influence behavior. In the case of fear extinction, the mPFCv appears to function by activating inhibitory GABAergic neurons within the amygdala (Berretta et al., 2005), and here the mPFCv may function by activating inhibitory neurons within the DRN.

The idea that the mPFCv exerts inhibitory control over structures that mediate fear and other stress-related processes might have some generality, and a reduction in this inhibitory control could be involved in mediating a number of stress-related clinical phenomena. Hypofunction in the mPFCv has been observed in a number of affective and anxiety disorders (Drevets, 2000; Davidson, 2002). Perceived control, or coping more generally, can buffer individuals against the negative impact of stress, and enhancement of mPFCv inhibitory control over limbic and brainstem stress-responsive structures may be an important mediating mechanism.

\section{References}

Amat J, Matus-Amat P, Watkins LR, Maier SF (1998) Escapable and inescapable stress differentially alter extracellular levels of 5-HT in the basolateral amygdala of the rat. Brain Res 812:113-120.

Amat J, Baratta MV, Paul E, Bland ST, Watkins LR, Maier SF (2005) Medial prefrontal cortex determines how stressor controllability affects behavior and dorsal raphe nucleus. Nat Neurosci 8:365-371.

Berretta S, Pantazopoulos H, Caldera M, Pantazopoulos P, Pare D (2005) Infralimbic cortex activation increases c-Fos expression in intercalated neurons of the amygdala. Neuroscience 132:943-953.

Castellano C, McGaugh JL (1990) Effects of post-training bicuculline and muscimol on retention: lack of state dependency. Behav Neural Biol 54:156-164.

Cardinal RN, Parkinson JA, Hall J, Everitt BJ (2002) Emotion and motivation: the role of the amygdala, ventral striatum, and prefrontal cortex. Neurosci Biobehav Rev 26:321-352.

Davidson RJ (2002) Anxiety and affective style: role of prefrontal cortex and amygdala. Biol Psychiatry 51:68-80.

Drevets WC (2000) Functional anatomical abnormalities in limbic and prefrontal cortical structures in major depression. Prog Brain Res 126:413-431.

Flexner LB, Goodman RH (1975) Studies on memory: inhibitors of protein synthesis also inhibit catecholamine synthesis. Proc Natl Acad Sci USA 72:4660-4663.
Graeff FG, Guimaraes FS, De Andrade TG, Deakin JF (1996) Role of 5-HT in stress, anxiety, and depression. Pharmacol Biochem Behav 54:129-141.

Grahn RE, Will MJ, Hammack SE, Maswood S, McQueen MB, Watkins LR, Maier SF (1999) Activation of serotonin-immunoreactive cells in the dorsal raphe nucleus in rats exposed to an uncontrollable stressor. Brain Res 826:35-43.

Inda MC, Delgado-Garcia JM, Carrion AM (2005) Acquisition, consolidation, reconsolidation, and extinction of eyelid conditioning responses require de novo protein synthesis. J Neurosci 25:2070-2080.

Jankowski MP, Sesack SR (2004) Prefrontal cortical projections to the rat dorsal raphe nucleus: ultrastructural features and associations with serotonin and gamma-aminobutyric acid neurons. J Comp Neurol 468:518-529.

Maier SF, Seligman MEP (1976) Learned helplessness: theory and evidence. J Exp Psychology Gen 105:3-46.

Maier SF, Watkins LR (1998) Stressor controllability, anxiety, and serotonin. Cognit Ther Res 22:595-613.

Maier SF, Watkins LR (2005) Stressor controllability and learned helplessness: the roles of the dorsal raphe nucleus, serotonin, and corticotropinreleasing factor. Neurosci Biobehav Rev 29:829-841.

Maier SF, Grahn RE, Watkins LR (1995) 8-OH-DPAT microinjected in the region of the dorsal raphe nucleus blocks and reverses the enhancement of fear conditioning and interference with escape produced by exposure to inescapable shock. Behav Neurosci 109:404-412.

Maswood S, Barter JE, Watkins LR, Maier SF (1998) Exposure to inescapable but not escapable shock increases extracellular levels of 5-HT in the dorsal raphe nucleus of the rat. Brain Res 783:115-120.

Miller EK, Cohen JD (2001) An integrative theory of prefrontal cortex function. Annu Rev Neurosci 24:167-202.

Morgan MA, Romanski LM, LeDoux JE (1993) Extinction of emotional learning: contribution of medial prefrontal cortex. Neurosci Lett 163:109-113.

Moye TB, Grau JW, Coon DJ, Maier SF (1981) Therapy and immunization of long-term analgesia in rats. Learn Motiv 12:133-148.

Ogawa T, Hayashi T, Kyoizumi S, Kusunoki Y, Nakachi K, MacPhee DG, Trosko JE, Kataoka K, Yorioka N (2004) Anisomycin downregulates gap-junctional intercellular communication via the p38 MAP-kinase pathway. J Cell Sci 117:2087-2096.

Peyron C, Petit JM, Rampon C, Jouvet M, Luppi PH (1998) Forebrain afferents to the rat dorsal raphe nucleus demonstrated by retrograde and anterograde tracing methods. Neuroscience 82:443-468.

Quirk GJ, Russo GK, Barron JL, Lebron K (2000) The role of ventromedial prefrontal cortex in the recovery of extinguished fear. J Neurosci 20:6225-6231.

Robbins TW (2000) From arousal to cognition: the integrative position of the prefrontal cortex. Prog Brain Res 126:469-483.

Routtenberg A, Rekart JL (2005) Post-translational protein modification as the substrate for long-lasting memory. Trends Neurosci 28:12-19.

Santini E, Ge H, Ren K, Pena de Ortiz S, Quirk GJ (2004) Consolidation of fear extinction requires protein synthesis in the medial prefrontal cortex. J Neurosci 24:5704-5710.

Seligman MEP, Maier SF (1967) Failure to escape traumatic shock. J Exp Psychol 74:1-9.

Sotres-Bayon F, Bush DE, LeDoux JE (2004) Emotional perseveration: an update on prefrontal-amygdala interactions in fear extinction. Learn Mem 11:525-535.

Takase LF, Nogueira MI, Baratta M, Bland ST, Watkins LR, Maier SF, Fornal CA, Jacobs BL (2004) Inescapable shock activates serotonergic neurons in all raphe nuclei of rat. Behav Brain Res 153:233-239.

Weiss JM (1968) Effects of coping responses on stress. J Comp Physiol Psychol 65:251-260.

Weiss JM, Simson PG (1986) Depression in an animal model: focus on the locus ceruleus. Ciba Found Symp 123:191-215.

Williams JL, Maier SF (1977) Transituational immunization and therapy of learned helplessness in the rat. J Exp Psychol Anim Behav Process 3:240-253.

Yin HH, Knowlton BJ, Balleine BW (2004) Lesions of dorsolateral striatum preserve outcome expectancy but disrupt habit formation in instrumental learning. Eur J Neurosci 19:181-189. 